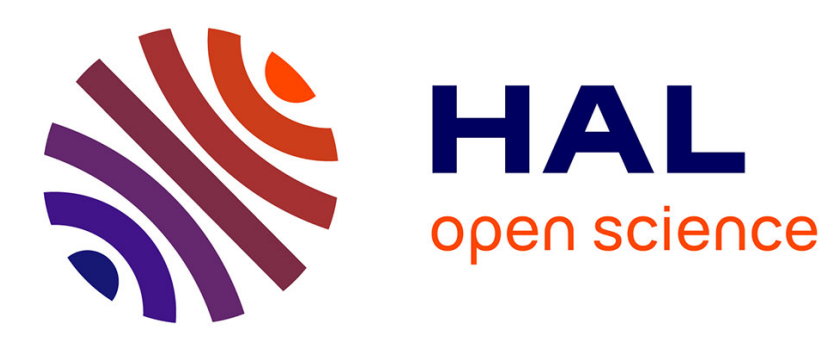

\title{
Balancing waveform relaxation for age-structured populations in a multilayer environment \\ Luca Gerardo-Giorda
}

\section{To cite this version:}

Luca Gerardo-Giorda. Balancing waveform relaxation for age-structured populations in a multilayer environment. 2007. hal-00191111

\section{HAL Id: hal-00191111 \\ https://hal.science/hal-00191111}

Preprint submitted on 23 Nov 2007

HAL is a multi-disciplinary open access archive for the deposit and dissemination of scientific research documents, whether they are published or not. The documents may come from teaching and research institutions in France or abroad, or from public or private research centers.
L'archive ouverte pluridisciplinaire HAL, est destinée au dépôt et à la diffusion de documents scientifiques de niveau recherche, publiés ou non, émanant des établissements d'enseignement et de recherche français ou étrangers, des laboratoires publics ou privés. 


\title{
Balancing waveform relaxation for age-structured populations in a multilayer environment
}

\author{
Luca Gerardo-GiorDA*
}

November 23, 2007

\begin{abstract}
In this paper we present a balancing domain decomposition method of Neumann-Neumann type for the numerical approximation of an age-structured population diffusing in a multilayer environment. We give a quasi optimal convergence result for the algorithm via Fourier analysis in a two-layers case, and we address the variational generalization to an arbitrary number of layers as well as its finite dimensional formulation. We illustrate our approach with some numerical results.
\end{abstract}

Keywords: Balancing domain decomposition, population dynamics, multilayer problems

\section{Introduction}

Over the last decades, in the field of population dynamics, a growing interest developed towards the modelisation of ecological problems such as the migration of populations or the spread of some epidemical disease. Modeling such problems involves several features of the population itself, and, in particular, empirical evidence suggests that both the spatial diffusion of individuals and the internal heterogeneity of the population have to be taken into account. In this direction, models for the diffusion of structured populations have been formulated and analyzed, focusing mainly on the case of age-structured populations. Especially in the case of ecological problems, attention must be paid to the spatial heterogeneities of the medium, whose peculiar characteristics reflect into the diffusion coefficients. In that order multi-layer models have been developed in the recent years (see [17] for biological motivations) and analyzed $([9,4,5])$, mostly from the theoretical point of view.

The mathematical problem describing the spreading of an age-structured population in a bounded region $\Omega \in$ $\mathbb{R}^{d}(d=1,2,3)$ consists in a reaction-diffusion equation for the population density, together with a given initial condition, an integral condition at age $a=0$, giving the newborns rate, and boundary conditions on $\partial \Omega$ depending on the specific features of the population and of the environment: an homogeneous Neumann boundary condition is used to model $\Omega$ as an isolated environment, while an homogeneous Dirichlet boundary condition models an hostile habitat at the boundary of $\Omega$. For an almost complete review of the results concerning existence, uniqueness and asymptotic behaviour of the solution of age-structured diffusion models, we refer the interested reader to the book by A. Okubo and S.A. Levin ([13], Sec.10). In this paper we present a balancing domain decomposition method of Neumann-Neumann type for the numerical approximation of an age-structured population diffusing in a multilayer environment.

The paper is organized as follows. In section 2 we state the multilayer problem, while in section 3 the method is introduced for a two-layers decomposition, and its convergence is analyzed at the continuous level by means of a

*Dipartimento di Matematica, Università di Trento, Via Sommarive 14, I-38050 Povo- Italy 
Fourier analysis. In section 4 we first give the variational formulation of the method in order to make it possible the generalization to an arbitrary number of layers, then we address the finite dimensional formulation of the method for the multilayer problem. Finally, in section 5, some numerical results illustrating the performance of the method conclude the paper.

\section{Diffusion in a multilayer environment and domain decomposition}

The spatial spread of an age-structured population in an isolated environment is commonly governed by a partial differential equation, with zero-flux boundary condition for the spatial domain. The variables involved are time, age and space, which will be denoted in the following by $t, a$ and $x$, respectively, whereas the unknown is the population density at time $t$ per unit volume and age, that we denote by $p(t, a, x)$. The spatial domain is $\Omega \subset \mathbb{R}^{d}(d=1,2,3)$, and we assume the age of the population to be bounded, i.e. there exists $a_{\dagger}>0$ such that $a \in\left[0, a_{\dagger}\right]$. The total population at time $t$ is then given by

$$
P(t)=\int_{\Omega} \int_{0}^{a_{\dagger}} p(t, a, x) d a d x .
$$

The population density $p(t, a, x)$ satisfies the following model problem.

Find $p(t, a, x) \in C\left(0, T ; L^{1}\left(0, a_{\dagger} ; H^{1}(\Omega)\right)\right)$ such that

$$
\begin{aligned}
\partial_{t} p+\partial_{a} p+\mu(a) p-\operatorname{div}(k(a, x) \nabla p)=g & \text { in }(0, T) \times\left(0, a_{\dagger}\right) \times \Omega \\
p(0, a, x)=p_{0}(a, x) & \text { in }\left(0, a_{\dagger}\right) \times \Omega \\
p(t, 0, x)=\int_{0}^{a_{\dagger}} \beta(a) p(t, a, x) d a & \text { in }(0, T) \times \Omega \\
\boldsymbol{n} \cdot(k(a, x) \nabla p)=0 & \text { on }(0, T) \times\left(0, a_{\dagger}\right) \times \partial \Omega .
\end{aligned}
$$

Here $\boldsymbol{n}$ denotes the outward normal to $\partial \Omega, g$ is a suitable forcing term, $\beta(a)$ is the age-specific fertility, and $\mu(a)$ is the age-specific mortality, such that

$$
\int_{0}^{a_{\dagger}} \mu(a) d a=+\infty
$$

We refer to [14] and references therein for issues concerning existence and uniqueness for the solution of problem (1). The probability of an individual to reach age $a$ is called survival probability, and is defined as

$$
\Pi(a)=\exp \left(-\int_{0}^{a} \mu(s) d s\right)
$$

Owing to (2), the survival probability at age $a_{\dagger}$ vanishes, ensuring that no individual exceeds the maximal age. One can then introduce a new variable

$$
u(t, a, x)=\frac{p(t, a, x)}{\Pi(a)}
$$

and a maternity function $m(a)=\beta(a) \Pi(a)$, so that $u(t, a, x)$ satisfies an equation similar to (1), but whose coefficients are no longer unbounded.

We consider here a population spreading in a stratified environment composed of $N$ layers, with zero flux boundary conditions. We refer the interested reader to [17] for issues concerning the motivations of such model. We let $\Omega=\bigcup_{j=1}^{N} \Omega_{j}$, as portrayed in Figure 1 , and we denote by $\Gamma_{j}=\partial \Omega_{j} \cap \partial \Omega_{j+1}(j=1, \ldots, N-1)$ the interface between the $j$-th and the $(j+1)$-th layer. 
We suppose that the age-specific fertility and the age-specific mortality depend only on the layer, while the diffusion coefficients depend both on the age and on the layer. On the interface $\Gamma_{j}$ one has to impose the continuity of the trace and the normal flux, thus the equation in the $j$-th layer reads

$$
\begin{array}{cl}
\partial_{t} u_{j}+\partial_{a} u_{j}-\operatorname{div}\left(k_{j}(a, x) \nabla u_{j}\right)=f_{j} & \text { in }(0, T) \times\left(0, a_{\dagger}\right) \times \Omega_{j} \\
u_{j}(0, a, x)=u_{0, j}(a, x) & \text { in }\left(0, a_{\dagger}\right) \times \Omega_{j} \\
u_{j}(t, 0, x)=\int_{0}^{a_{\dagger}} m_{j}(a) u_{j}(t, a, x) d a & \text { in }(0, T) \times \Omega_{j} \\
\boldsymbol{n}_{j} \cdot\left(k_{j}(a, x) \nabla u_{j}\right)=0 & \text { on }(0, T) \times\left(0, a_{\dagger}\right) \times\left(\partial \Omega \cap \partial \Omega_{j}\right) \\
u_{j}(t, a, x)=u_{j-1}(t, a, x) & \text { on }(0, T) \times\left(0, a_{\dagger}\right) \times \Gamma_{j-1} \\
u_{j}(t, a, x)=u_{j+1}(t, a, x) & \text { on }(0, T) \times\left(0, a_{\dagger}\right) \times \Gamma_{j} \\
\boldsymbol{n}_{j} \cdot\left(k_{j} \nabla u_{j}\right)=\boldsymbol{n}_{j} \cdot\left(k_{j-1} \nabla u_{j-1}\right) & \text { on }(0, T) \times\left(0, a_{\dagger}\right) \times \Gamma_{j-1} \\
\boldsymbol{n}_{j} \cdot\left(k_{j} \nabla u_{j}\right)=\boldsymbol{n}_{j} \cdot\left(k_{j+1} \nabla u_{j+1}\right) & \text { on }(0, T) \times\left(0, a_{\dagger}\right) \times \Gamma_{j}
\end{array}
$$

The fertility functions $\beta_{j}(a)$ are positive function of age: we assume it is measurable and essentially bounded, namely there exists $\beta_{+}>0$ such that

$$
0 \leq \beta_{j}(a)<\beta_{+} .
$$

Under this assumption $m_{j}(a) \in L^{\infty}\left(0, a_{\dagger}\right)$, and we assume that the diffusion coefficients are such that, for all $j=1, \ldots, N, k_{j}(a, x) \in L^{\infty}\left(\left(0, a_{\dagger}\right) \times \Omega\right)$, and $0<k_{0} \leq k_{j}(a, x) \leq k_{+}$. We refer to [4] for existence and uniqueness of the solution of (3), and we point out that a non-overlapping domain decomposition procedure to solve equation (3) is naturally induced by the physics of the problem.

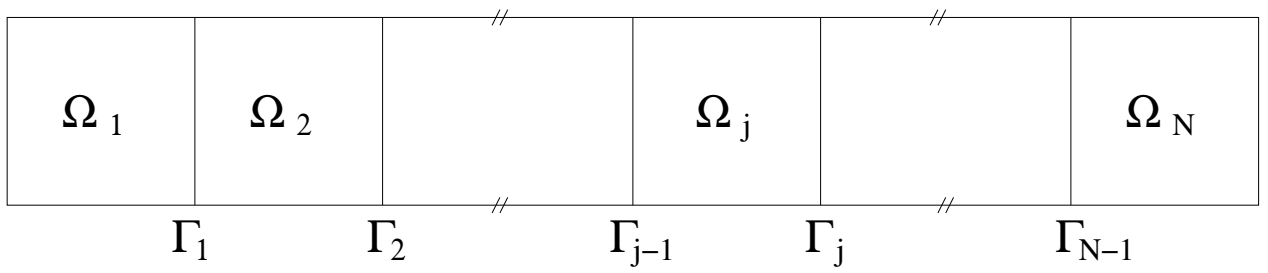

Figure 1: The multilayer domain $\Omega=\bigcup_{j=1}^{N} \Omega_{j}$

\section{$2.1 \quad$ Time discretization}

Classical approaches to the numerical solution of (3) integrate along the characteristics in age and time (see for instance $[7,8,12])$. However, the presence of different time scales in the dynamics suggests the use of different steps in the discretization of time and age (see [1,3]). Let us consider a discretization of the interval $(0, T)$ into $N_{t}$ subintervals of lenght $\Delta t=T / N_{t}$ (for simplicity we consider a uniform discretization). For equation (3) we advance in time by means of a backward Euler scheme, where the initial condition in age is computed at the previous time step. At each time step, we solve the coupled parabolic problem in age and space: 
Given $u_{j}^{0}(a, x)=u_{0, j}(a, x)$, find, for $n \geq 1, u_{j}^{n} \in L^{1}\left(0, a_{\dagger} ; H^{1}(\Omega)\right)(j=1, \ldots, N)$ such that

$$
\begin{array}{cl}
\partial_{a} u_{j}^{n}-\operatorname{div}\left(k_{j}(a, x) \nabla u_{j}^{n}\right)+\frac{1}{\Delta t} u_{j}^{n}=f_{j}+\frac{1}{\Delta t} u_{j}^{n-1} & \text { in }\left(0, a_{\dagger}\right) \times \Omega_{j} \\
u_{j}^{n}(0, x)=\int_{0}^{a_{\dagger}} m_{j}(a) u_{j}^{n-1}(a, x) d a & \text { in } \Omega_{j} \\
\boldsymbol{n}_{j} \cdot\left(k_{j}(a, x) \nabla u_{j}\right)=0 & \text { on }\left(0, a_{\dagger}\right) \times\left(\partial \Omega \cap \partial \Omega_{j}\right) \\
u_{j}^{n}(a, x)=u_{j-1}^{n}(a, x) & \text { on }\left(0, a_{\dagger}\right) \times \Gamma_{j-1} \\
u_{j}^{n}(a, x)=u_{j+1}^{n}(a, x) & \text { on }\left(0, a_{\dagger}\right) \times \Gamma_{j} \\
\boldsymbol{n}_{j} \cdot\left(k_{j} \nabla u_{j}^{n}\right)=\boldsymbol{n}_{j} \cdot\left(k_{j-1} \nabla u_{j-1}^{n}\right) & \text { on }\left(0, a_{\dagger}\right) \times \Gamma_{j-1} \\
\boldsymbol{n}_{j} \cdot\left(k_{j} \nabla u_{j}^{n}\right)=\boldsymbol{n}_{j} \cdot\left(k_{j+1} \nabla u_{j+1}^{n}\right) & \text { on }\left(0, a_{\dagger}\right) \times \Gamma_{j} .
\end{array}
$$

\section{A Balancing Neumann-Neumann waveform relaxation method}

In each time slab $\left(t^{n-1}, t^{n}\right)$, the interface continuities of problem (5) are enforced by means of a waveform relaxation algorithm of Neumann-Neumann type. Since the diffusion coefficients a priori differ from one layer to the other, the natural choice is to extend to such situation the Balancing Neumann-Neumann method designed for pure elliptic problems ([10]).

For sake of simplicity in presentation, we give here the two-domain formulation of the algorithm. We set $\Omega=\Omega_{1} \cup \Omega_{2}$, we denote the interface between the two subdomains by $\Gamma=\partial \Omega_{1} \cap \partial \Omega_{2}$, and the space of traces on $\Gamma$ of functions in $H^{1}(\Omega)$ by $\Lambda=H^{1 / 2}(\Gamma)$. The algorithm reads as follows.

Step 1: At each time step, given an initial value $\lambda^{n, 0} \in L^{1}\left(\left(0, a_{\dagger}\right) ; \Lambda\right)$, solve:

$$
\begin{cases}\partial_{a} u_{j}^{n, l+1}-\operatorname{div}\left(k_{j}(a, x) \nabla u_{j}^{n, l+1}\right)+\frac{1}{\Delta t} u_{j}^{n, l+1}=f_{j}+\frac{1}{\Delta t} u_{j}^{n-1} & \text { in } \Omega_{j} \\ u_{j}^{n, l+1}=\lambda^{n, l} & \text { on }\left(0, a_{\dagger}\right) \times \Gamma \\ u_{j}^{n, l+1}(0, x)=u_{j}^{n}(0, x) & \end{cases}
$$

Step 2: Solve

$$
\begin{cases}\partial_{a} \psi_{j}^{n, l+1}-\operatorname{div}\left(k_{j}(a, x) \nabla \psi_{j}^{n, l+1}\right)+\frac{1}{\Delta t} \psi_{j}^{n, l+1}=0 & \text { in } \Omega_{j} \\ k_{j} \mathbf{n}_{j} \cdot \nabla \psi_{j}^{n, l+1}=\frac{k_{j}}{k_{1}+k_{2}}\left(k_{1} \mathbf{n}_{1} \cdot \nabla u_{1}^{n, l+1}+k_{2} \mathbf{n}_{2} \cdot \nabla u_{2}^{n, l+1}\right) & \text { on }\left(0, a_{\dagger}\right) \times \Gamma \\ \psi_{j}^{n, l+1}(0, x)=0 & \end{cases}
$$

Step 3: Set

$$
\lambda^{l+1}=\lambda^{n, l}-\vartheta\left(\frac{k_{1}}{k_{1}+k_{2}} \psi_{1}^{n, l+1}-\frac{k_{2}}{k_{1}+k_{2}} \psi_{2}^{n, l+1}\right)_{\mid\left(0, a_{\dagger}\right) \times \Gamma}
$$

and iterate until convergence. 


\subsection{Convergence analysis}

Since the problem in the time slab $\left(t^{n-1}, t^{n}\right)$ is linear, it is enough to prove convergence to the zero solution of the homogeneous problem. For sake of readability, we drop, in the rest of this section, any index referring to time discretisation, and we set $\gamma=\frac{1}{\Delta t}$. We prove convergence, via a Fourier analysis, in the case of an infinite spatial domain $\Omega=\mathbb{R}^{2}$, decomposed into $\Omega_{1}=\mathbb{R}^{-} \times \mathbb{R}$ and $\Omega_{2}=\mathbb{R}^{+} \times \mathbb{R}$. We assume that the diffusion coefficients are constant in each subdomain, and we assume that the solutions $u_{j}(a, x, y)(j=1,2)$ decay at $x \rightarrow \pm \infty$. The local problems are then set in $\left(0, a_{\dagger}\right) \times \Omega_{1}$ and $\left(0, a_{\dagger}\right) \times \Omega_{2}$ :

$$
\begin{aligned}
\partial_{a} u_{j}-\operatorname{div}\left(k_{j} \nabla u_{j}\right)+\gamma u_{j}=0 & \text { in }\left(0, a_{\dagger}\right) \times \Omega_{j} \\
u_{j}(0, x, y)=0 & \text { in } \Omega_{j} \\
u_{1}(a, 0, y)=u_{2}(a, 0, y) & \text { on }\left(0, a_{\dagger}\right) \times \mathbb{R} \\
\boldsymbol{n}_{1} \cdot\left(k_{1} \nabla u_{1}\right)=\boldsymbol{n}_{2} \cdot\left(k_{2} \nabla u_{2}\right) & \text { on }\left(0, a_{\dagger}\right) \times \mathbb{R}
\end{aligned}
$$

We are in the position to prove the following result.

Proposition 3.1 In the case where the plane $\mathbb{R}^{2}$ is decomposed into the left and the right half-planes, and the diffusion coefficients are constant upon each subdomain, the waveform relaxation algorithm defined in (6)-(8) applied to (9) converges, provided

$$
0<\vartheta \leq \vartheta^{*}=2 \frac{\left(1+\eta^{2}\right)(1+\eta)^{2}}{\left(1+\eta^{2}\right)^{2}+(1+\eta)^{2}},
$$

where $\eta \in(0,1)$ is the ratio between the diffusion coefficients.

Proof We prove the result via Fourier analysis. We assume that all functions are extended by 0 for $a<0$ and for $a>a_{+}$, we perform a partial Fourier transform in the $a$ and $y$ variable, that we denote with $\mathcal{F}$, and we call $\omega$ and $\xi$, respectively, the corresponding dual variables. The transform $\mathcal{F}$ is defined as

$$
\mathcal{F}: \mathbf{u}(a, x, y) \rightarrow \widehat{\mathbf{u}}(\omega, x, \xi)=\int_{\mathbb{R}} \mathbf{u}(a, x, y) e^{-i(\omega a+\xi y)} d a d y,
$$

and, inside each subdomain, we seek for the solution of

$$
\left(-k_{j} \partial_{x x}+k_{j} \xi^{2}-i \omega+\gamma\right) \widehat{u}_{j}(\omega, x, \xi)=0 .
$$

The solutions $\widehat{u}_{1}^{l+1}(\omega, x, \xi)$ and $\widehat{u}_{2}^{l+1}(\omega, x, \xi)$ of $(6)$ are given by

$$
\widehat{u}_{1}^{l+1}(\omega, x, \xi)=\widehat{\lambda}^{l}(\omega, \xi) e^{\sqrt{\frac{k_{1} \xi^{2}+\gamma-i \omega}{k_{1}}} x} \widehat{u}_{2}^{l+1}(\omega, x, \xi)=\widehat{\lambda}^{l}(\omega, \xi) e^{-\sqrt{\frac{k_{2} \xi^{2}+\gamma-i \omega}{k_{2}}} x},
$$

where $\widehat{\lambda}^{l}(\omega, \xi)$ denotes the Fourier transform of $\lambda^{l}(a, y)$. Denoting by $g^{l+1}$ the interface value $g^{l+1}=k_{1} \mathbf{n}_{1}$. $\nabla u_{1}^{l+1}+k_{2} \mathbf{n}_{2} \cdot \nabla u_{2}^{l+1}$, the solutions $\widehat{\psi}_{1}^{l+1}(\omega, x, \xi)$ and $\widehat{\psi}_{2}^{l+1}(\omega, x, \xi)$ of $(7)$ are given by

$$
\begin{aligned}
& \widehat{\psi}_{1}(\omega, x, \xi)^{l+1}=g^{l+1} \frac{1}{\sqrt{k_{1}} \sqrt{k_{1} \xi^{2}+\gamma-i \omega}} e^{\sqrt{\frac{k_{1} \xi^{2}+\gamma-i \omega}{k_{1}} x}} \\
& \widehat{\psi}_{2}(\omega, x, \xi)^{l+1}=g^{l+1} \frac{1}{\sqrt{k_{2}} \sqrt{k_{2} \xi^{2}+\gamma-i \omega}} e^{-\sqrt{\frac{k_{1} \xi^{2}+\gamma-i \omega}{k_{1}}} x} .
\end{aligned}
$$


Thus we have in (8):

$$
\begin{aligned}
\frac{k_{1}}{k_{1}+k_{2}} \widehat{\psi}_{1}(\omega, 0, \xi)^{l+1}+\frac{k_{2}}{k_{1}+k_{2}} \widehat{\psi}_{2}(\omega, 0, \xi)^{l+1}= \\
\quad=\left(\left[\frac{k_{1}}{k_{1}+k_{2}}\right]^{2} \frac{1}{\sqrt{k_{1}} \sqrt{k_{1} \xi^{2}+\gamma-i \omega}}+\left[\frac{k_{2}}{k_{1}+k_{2}}\right]^{2} \frac{1}{\sqrt{k_{2}} \sqrt{k_{2} \xi^{2}+\gamma-i \omega}}\right) g^{l+1}
\end{aligned}
$$

Owing to (11),

$$
\begin{aligned}
g^{l+1}=k_{1} \mathbf{n}_{1} \cdot \nabla u_{1}^{l+1}+ & k_{2} \mathbf{n}_{2} \cdot \nabla u_{2}^{l+1}= \\
& =\left(\sqrt{k_{1}} \sqrt{k_{1} \xi^{2}+\gamma-i \omega}+\sqrt{k_{2}} \sqrt{k_{2} \xi^{2}+\gamma-i \omega}\right) \widehat{\lambda}^{l}(\omega, \xi),
\end{aligned}
$$

and, letting $N_{1}=\left[\frac{k_{1}}{k_{1}+k_{2}}\right]^{2}, N_{2}=\left[\frac{k_{2}}{k_{1}+k_{2}}\right]^{2}$, the iterative mapping reads

$$
\widehat{\lambda}^{l+1}(\omega, \xi)=A(\omega, \xi, \vartheta) \widehat{\lambda}^{l}(\omega, \xi)
$$

where

$$
\begin{aligned}
& A(\omega, \xi, \vartheta)= \\
& \quad=1-\vartheta\left\{N_{1}\left(1+\frac{\sqrt{k_{2}^{2} \xi^{2}+k_{2} \gamma-i k_{2} \omega}}{\sqrt{k_{1}^{2} \xi^{2}+k_{1} \gamma-i k_{1} \omega}}\right)+N_{2}\left(1+\frac{\sqrt{k_{1}^{2} \xi^{2}+k_{1} \gamma-i k_{1} \omega}}{\sqrt{k_{2}^{2} \xi^{2}+k_{2} \gamma-i k_{2} \omega}}\right)\right\} .
\end{aligned}
$$

With no loss of generality, we assume $k_{1} \leq k_{2}$, and we let $z(\omega, \xi)=\sqrt{\frac{k_{2}^{2} \xi^{2}+k_{2} \gamma-i k_{2} \omega}{k_{1}^{2} \xi^{2}+k_{1} \gamma-i k_{1} \omega}}$. It is then easy to see that, for any $(\omega, \xi) \in \mathbb{R}^{2}$,

$$
1<|z(\omega, \xi)|=\left[\frac{\left(k_{2}^{2} \xi^{2}+k_{2} \gamma\right)^{2}+\left(k_{2} \omega\right)^{2}}{\left(k_{1}^{2} \xi^{2}+k_{1} \gamma\right)^{2}+\left(k_{1} \omega\right)^{2}}\right]^{1 / 4} \leq \frac{k_{2}}{k_{1}},
$$

and the waveform relaxation algorithm is convergent if

$$
|A(\omega, \xi, \vartheta)|=\left|1-\vartheta\left[N_{1}(1+z(\omega, \xi))+N_{2}\left(1+[z(\omega, \xi)]^{-1}\right)\right]\right|<1 .
$$

We have, as $[z(\omega, \xi)]^{-1}=|z(\omega, \xi)|^{-2} \bar{z}(\omega, \xi)$,

$$
\begin{aligned}
& \operatorname{Re} \mathrm{A}(\mathrm{z}(\omega, \xi), \vartheta)=1-\vartheta\left(N_{1}+N_{2}\right)-\vartheta\left(N_{1}+\frac{N_{2}}{|z(\omega, \xi)|^{2}}\right) \operatorname{Re} \mathrm{z}(\omega, \xi) \\
& \operatorname{Im} \mathrm{A}(\mathrm{z}(\omega, \xi), \vartheta)=-\vartheta\left(N_{1}-\frac{N_{2}}{|z(\omega, \xi)|^{2}}\right) \operatorname{Im} \mathrm{z}(\omega, \xi) .
\end{aligned}
$$


Since $\operatorname{Re} \mathrm{z}(\omega, \xi)>0$, and $1-\vartheta\left(N_{1}+N_{2}\right)>0$, we can estimate $|A(z(\omega, \xi), \vartheta)|^{2}$ by

$$
\begin{gathered}
|A(z(\omega, \xi), \vartheta)|^{2} \leq\left[1-\vartheta\left(N_{1}+N_{2}\right)\right]^{2}+\vartheta^{2}\left[N_{1}^{2}+\frac{N_{2}^{2}}{|z(\omega, \xi)|^{4}}\right]|z(\omega, \xi)|^{2} \\
\quad+2 \vartheta^{2} \frac{N_{1} N_{2}}{|z(\omega, \xi)|^{2}}\left[(\operatorname{Rez}(\omega, \xi))^{2}-(\operatorname{Imz}(\omega, \xi))^{2}\right] \\
=\left[1-\vartheta\left(N_{1}+N_{2}\right)\right]^{2}+\vartheta^{2}\left[N_{1}^{2}+\frac{N_{2}^{2}}{|z(\omega, \xi)|^{4}}\right]|z(\omega, \xi)|^{2} \\
\quad+2 \vartheta^{2} \frac{N_{1} N_{2}}{|z(\omega, \xi)|^{2}} \operatorname{Re}[\mathrm{z}(\omega, \xi)]^{2} \\
\leq\left[1-\vartheta\left(N_{1}+N_{2}\right)\right]^{2}+\vartheta^{2}\left[N_{1}^{2}|z(\omega, \xi)|^{2}+\frac{N_{2}^{2}}{|z(\omega, \xi)|^{2}}+2 N_{1} N_{2}\right] \\
=\left[1-\vartheta\left(N_{1}+N_{2}\right)\right]^{2}+\vartheta^{2}\left[N_{1}|z(\omega, \xi)|+\frac{N_{2}}{|z(\omega, \xi)|}\right]^{2} .
\end{gathered}
$$

Owing to (15), and setting $\eta:=\frac{k_{1}}{k_{2}}$, a simple algebraic manipulation provides

$$
\begin{aligned}
|A(z(\omega, \xi), \vartheta)|^{2} & \leq\left[1-\vartheta \frac{1+\eta^{2}}{(1+\eta)^{2}}\right]^{2}+\left[\vartheta \frac{1+\eta}{(1+\eta)^{2}}\right]^{2} \\
& =1-2 \frac{1+\eta^{2}}{(1+\eta)^{2}} \vartheta+\frac{\left(1+\eta^{2}\right)^{2}+(1+\eta)^{2}}{(1+\eta)^{4}} \vartheta^{2}=\Phi(\eta, \vartheta) .
\end{aligned}
$$

The function $\Phi(\eta, \vartheta)$ is strictly positive. As the level set $\Phi(\eta, \vartheta)=1$ consists in the functions $\vartheta=0$ and $\vartheta=2 \frac{\left(1+\eta^{2}\right)(1+\eta)^{2}}{\left(1+\eta^{2}\right)^{2}+(1+\eta)^{2}}$, and, for any fixed $\bar{\eta} \in(0,1), \Phi(\bar{\eta}, \vartheta)$ is a convex function of $\vartheta$, the convergence condition (16) is satisfied whenever (see Figure 2)

$$
0<\vartheta<2 \frac{\left(1+\eta^{2}\right)(1+\eta)^{2}}{\left(1+\eta^{2}\right)^{2}+(1+\eta)^{2}}
$$

Remark 3.1 The waveform relaxation procedure (6)-(8) points at solving the interface equation

$$
\mathcal{S} u_{\Gamma}=\chi \quad \text { on }\left(0, a_{\dagger}\right) \times \Gamma
$$

where $u_{\Gamma} \in L^{1}\left(0, a_{\dagger} ; H_{00}^{1 / 2}(\Gamma)\right)$ is the restriction of the exact solution to $\left(0, a_{\dagger}\right) \times \Gamma$, and where $\mathcal{S}: L^{1}\left(0, a_{\dagger} ; H_{00}^{1 / 2}(\Gamma)\right) \rightarrow$ $L^{1}\left(0, a_{\dagger} ; H^{-1 / 2}(\Gamma)\right)$ is the Steklov-Poincaré operator, whose action can be expressed in terms of its Fourier transform in the $a$ and $y$ directions as

$$
\mathcal{S} u_{\Gamma}=\mathcal{F}^{-1}\left(\left[\Sigma_{1}(\omega, \xi)+\Sigma_{2}(\omega, \xi)\right] \widehat{u}_{\Gamma}(\omega, \xi)\right) \quad u_{\Gamma} \in L^{1}\left(0, a_{\dagger} ; H_{00}^{1 / 2}(\Gamma)\right),
$$




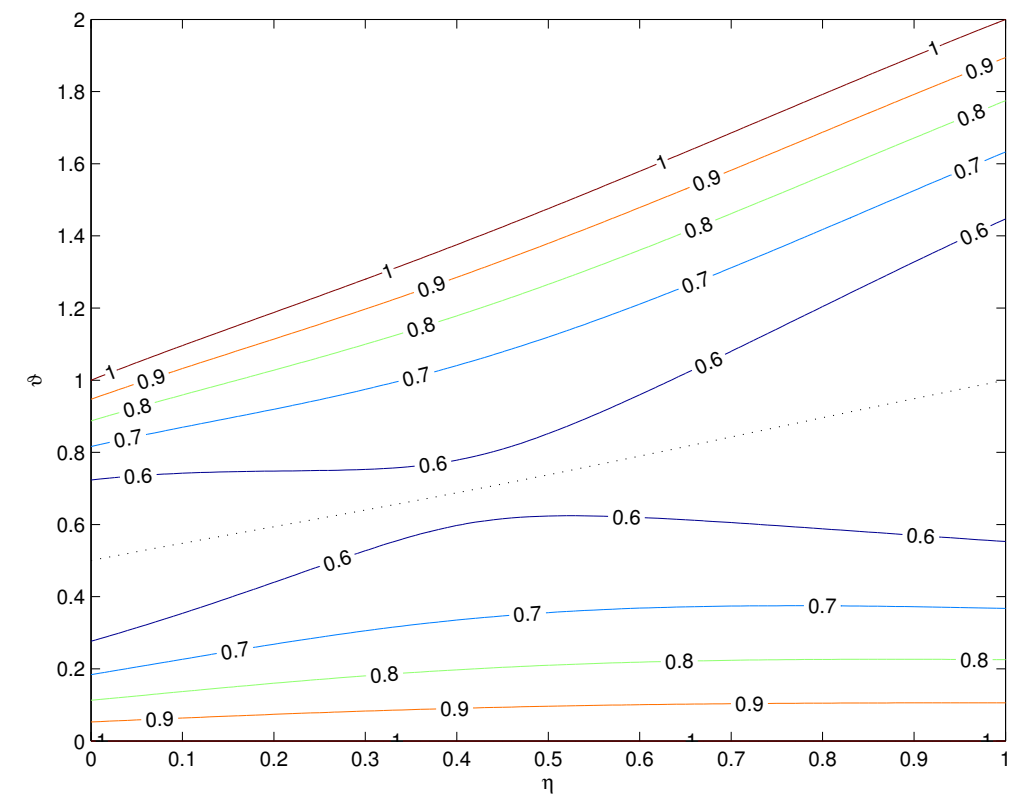

Figure 2: Contour levels of the function $\Phi(\eta, \vartheta)$.

$\Sigma_{1}(\omega, \xi)$ and $\Sigma_{2}(\omega, \xi)$ denoting the symbols of the local Steklov-Poincaré operators in $\left(0, a_{\dagger}\right) \times \Omega_{1}$ and $\left(0, a_{\dagger}\right) \times \Omega_{2}$, respectively. The waveform relaxation procedure (6)-(8) amounts to solve the Richardson iterative procedure preconditioned by means of the operator $\mathcal{T}: L^{1}\left(0, a_{\dagger} ; H^{-1 / 2}(\Gamma)\right) \rightarrow L^{1}\left(0, a_{\dagger} ; H^{1 / 2}(\Gamma)\right)$, defined as

$$
\mathcal{T} g=\mathcal{F}^{-1}\left(\left[\frac{k_{1}}{k_{1}+k_{2}}\left[\Sigma_{1}(\omega, \xi)\right]^{-1} \frac{k_{1}}{k_{1}+k_{2}}+\frac{k_{2}}{k_{1}+k_{2}}\left[\Sigma_{2}(\omega, \xi)\right]^{-1} \frac{k_{2}}{k_{1}+k_{2}}\right] g\right)
$$

for all $g \in L^{1}\left(0, a_{\dagger} ; H^{-1 / 2}(\Gamma)\right)$.

An immediate consequence of the preceeding argument is given by the following proposition.

Proposition 3.2 In the case where the plane $\mathbb{R}^{2}$ is decomposed into the left and the right half-planes, and the coefficients are constant upon each subdomain and equal, the waveform relaxation Neumann-Neumann algorithm (6)-(8) is an exact preconditioner for the Steklov-Poincaré operator.

Proof Let $u_{\Gamma} \in L^{1}\left(0, a_{\dagger} ; H_{00}^{1 / 2}(\Gamma)\right)$ be the restriction of the exact solution to the interface $\left(0, a_{\dagger}\right) \times \Gamma$, and let $k=k_{1}=k_{2}$. From the above argument, the Steklov-Poincaré operator can be split as $\mathcal{S}=\mathcal{S}_{1}+\mathcal{S}_{2}$, and the action of $\mathcal{S}_{1}$ and $\mathcal{S}_{2}$ can be expressed in terms of their Fourier transform in the $a$ and $y$ directions.

The symbols of the two operators are

$$
\Sigma_{1}(\omega, \xi)=\Sigma_{2}(\omega, \xi)=\sqrt{k^{2} \xi^{2}+k \gamma-i k \omega},
$$

thus

$$
\mathcal{F}\left(\mathcal{S}\left(u_{\Gamma}\right)\right)=\Sigma_{1}(\omega, \xi) \widehat{u}_{\Gamma}(\omega, \xi)+\Sigma_{2}(\omega, \xi) \widehat{u}_{\Gamma}(\omega, \xi)=2 \sqrt{k^{2} \xi^{2}+k \gamma-i k \omega} \widehat{u}_{\Gamma}(\omega, \xi) .
$$

In a similar way, it can be seen that the symbol of the Neumann to Dirichlet operator is given by

$$
\mathcal{F}(\mathcal{T}(g))=\left[\frac{1}{4} \frac{1}{\sqrt{k^{2} \xi^{2}+k \gamma-i k \omega}}+\frac{1}{4} \frac{1}{\sqrt{k^{2} \xi^{2}+k \gamma-i k \omega}}\right] \widehat{g}(\omega, \xi) .
$$


Then, we have

$$
\mathcal{F}\left([\mathcal{T} \circ \mathcal{S}] u_{\Gamma}\right)=\frac{1}{2} \frac{1}{\sqrt{k^{2} \xi^{2}+k \gamma-i k \omega}}\left(2 \sqrt{k^{2} \xi^{2}+k \gamma-i k \omega} \widehat{u}_{\Gamma}(\omega, \xi)\right)=\widehat{u}_{\Gamma}(\omega, \xi),
$$

namely $T \circ S=I d$, and the preconditioner is exact.

\section{Variational formulation and finite dimensional approximation}

In this section we go back to the multilayer formulation (5). First, we give a variational formulation of the single layer problem. Since, for all $n \geq 0$, the solution $u^{n} \in L^{1}\left(0, a_{\dagger} ; H^{1}(\Omega)\right)$, we choose as test functions any $v \in H^{1}(\Omega)$, and we integrate (5) over the spatial domain $\Omega$. The problem can then be recast as follows.

Given $u^{0} \in L^{1}\left(0, a_{\dagger} ; V_{h}\right)$, for all $n=1, \ldots, N_{t}$, find $u^{n} \in L^{1}\left(0, a_{\dagger} ; H^{1}(\Omega)\right)$ such that

$$
\left\{\begin{array}{l}
\frac{d}{d a}\left\langle u^{n}, v\right\rangle+A\left(a ; u^{n}, v\right)=\left(f^{n}, v\right) \\
u^{n}(0, x)=\int_{0}^{a_{\dagger}} m(a) u^{n-1}(a, x) d a,
\end{array} \quad \forall v \in H^{1}(\Omega)\right.
$$

where, for sake of readability, we set with a little abuse of notations $f^{n}=f\left(t^{n}\right)+\frac{1}{\Delta t} u^{n-1}$, where $\langle\cdot, \cdot\rangle$ is the duality pairing between $H^{1}(\Omega)$ and $H^{-1}(\Omega),(\cdot, \cdot)$ is the inner product in $L^{2}(\Omega)$, and the bilinear form $A(a ; u, v)$ is given by

$$
A(a ; \psi, \varphi)=\int_{\Omega} k(a, x) \nabla \psi \cdot \nabla \varphi d x+\frac{1}{\Delta t}(\psi, \varphi) \quad \forall \psi, \varphi \in H^{1}(\Omega) .
$$

The boundedness hypoteses on the coefficients of the problem entail continuity and coerciveness for the bilinear form $A(a ; \cdot, \cdot)$, and problem (19) has a unique solution $u^{n} \in L^{2}\left(0, a_{\dagger} ; H^{1}(\Omega)\right) \cap L^{\infty}\left(0, a_{\dagger} ; L^{2}(\Omega)\right.$ ) (see [11]).

We define the global interface as $\Gamma=\bigcup_{j=1}^{N-1} \Gamma_{j}$. We denote by $V_{j}=H^{1}\left(\Omega_{j}\right)$ the restrictions of $H^{1}(\Omega)$ to $\Omega_{j}$, by $\Lambda_{j}=L^{1}\left(0, a_{\dagger} ; H^{1 / 2}\left(\Gamma_{j}\right)\right)$ the space of traces upon $\Gamma_{j}$ of functions of $L^{1}\left(0, a_{\dagger} ; H^{1}(\Omega)\right)$, and by $\Lambda=\oplus_{j=1}^{N-1} \Lambda_{j}$ the global trace space, endowed with the norm $\|\boldsymbol{\lambda}\|_{\Lambda}=\sup _{j=1, \ldots, N-1}\left\|\boldsymbol{\lambda}_{j}\right\|_{\Lambda_{j}}$. At each time step, the coupled problem reads as follows:

Find $u_{j}^{n} \in L^{1}\left(0, a_{\dagger} ; H^{1}\left(\Omega_{j}\right)\right)(j=1, \ldots, N)$ such that

$$
\left\{\begin{array}{rrr}
\frac{d}{d a}<u_{j}^{n}, v_{j}>+A_{j}\left(a ; u_{j}^{n}, v_{j}\right)=\left(f_{j}^{n}, v_{j}\right) & & \forall v_{j} \in V_{j} \\
u_{j}^{n}=u_{j-1}^{n} \quad(j=2, \ldots, N) & \text { on }\left(0, a_{\dagger}\right) \times \Gamma_{j-1} \\
u_{j}^{n}=u_{j+1}^{n} \quad(j=1, \ldots, N-1) & \text { on }\left(0, a_{\dagger}\right) \times \Gamma_{j} \\
\frac{d}{d a}<u_{j+1}^{n}, R_{j+1} \mu>+A_{j+1}\left(a ; u_{j+1}^{n}, R_{j+1} \mu\right)= & \\
& =\left(f^{n}, R_{j+1} \mu\right)+\left(f^{n}, R_{j} \mu\right)-\frac{d}{d a}<u_{j}^{n}, R_{j} \mu>-A_{j}\left(a ; u_{j}^{n}, R_{j} \mu\right), & \\
\frac{d}{d a}<u_{j}^{n}, R_{j} \mu>+A_{j}\left(a ; u_{j}^{n}, R_{j} \mu\right)=\left(f^{n}, R_{j} \mu\right) & \\
& &
\end{array}\right.
$$


where $A_{j}(a ; \cdot, \cdot)$ denotes the restriction of the bilinear form $A(a ; \cdot, \cdot)$ to $\Omega_{j}$, whereas $R_{j} \mu$ denotes any possible extension of $\mu$ to $\Omega_{j}(j=1, \ldots, N)$.

The Balancing Neumann-Neumann waveform relaxation reads then, at each time step:

Step 1: Given an initial value $\lambda^{0} \in \Lambda$, solve, for $j=1, \ldots, N$ :

$$
\begin{cases}\frac{d}{d a}<u_{j}^{n, l+1}, v_{j}>+A_{j}\left(a ; u_{j}^{n, l+1}, v_{j}\right)=\left(f_{j}^{n}, v_{j}\right) & \forall v_{j} \in V_{j} \\ u_{j}^{n, l+1}=\lambda_{j}^{n, l} \quad(j=1, \ldots, N-1) & \text { on }\left(0, a_{\dagger}\right) \times \Gamma_{j} \\ u_{j}^{n, l+1}=\lambda_{j-1}^{n, l} \quad(j=2, \ldots, N) & \text { on }\left(0, a_{\dagger}\right) \times \Gamma_{j-1} \\ u_{j}^{n, l+1}(0, x)=u_{j}^{0}(x) & \end{cases}
$$

Step 2: Solve, for $j=1, \ldots, N$ :

$$
\left\{\begin{array}{cc}
\frac{d}{d a}<\psi_{j}^{n, l+1}, v_{j}>+A_{j}\left(a ; \psi_{j}^{n, l+1}, v_{j}\right)=0 & \forall v_{j} \in V_{j} \\
\frac{d}{d a}<\psi_{j}^{n, l+1}, R_{j} \mu>+A_{j}\left(a ; \psi_{j}^{n, l+1}, R_{j} \mu\right)= & \forall \mu \in \Lambda_{j} \\
=\frac{d}{d a}<u_{j}^{n, l+1}, R_{j} \mu>+\frac{d}{d a}<u_{j+1}^{n, l+1}, R_{j+1} \mu> & (j=1, \ldots, N-1) \\
\quad+A_{j}\left(a ; u_{j}^{n, l+1}, R_{j} \mu\right)+A_{j+1}\left(a ; u_{j+1}^{n, l+1}, R_{j+1} \mu\right)-\left(f^{n}, R_{j+1} \mu\right)-\left(f^{n}, R_{j} \mu\right) \\
\frac{d}{d a}<\psi_{j}^{n, l+1}, R_{j} \mu>+A_{j}\left(a ; \psi_{j}^{n, l+1}, R_{j} \mu\right)= & \forall \mu \in \Lambda_{j-1} \\
=-\frac{d}{d a}<u_{j-1}^{n, l+1}, R_{j-1} \mu>-\frac{d}{d a}<u_{j}^{n, l+1}, R_{j} \mu> & (j=2, \ldots, N) \\
-A_{j-1}\left(a ; u_{j-1}^{n, l+1}, R_{j-1} \mu\right)-A_{j}\left(a ; u_{j}^{n, l+1}, R_{j} \mu\right)+\left(f^{n}, R_{j} \mu\right)+\left(f^{n}, R_{j-1} \mu\right) \\
\psi_{j}^{n, l+1}(0, x)=0 .
\end{array}\right.
$$

Step 3: Set, for $j=1, \ldots, N$

$$
\lambda_{j}^{n, l+1}=\lambda_{j}^{n, l}-\vartheta\left(\frac{k_{j}}{k_{j}+k_{j+1}} \psi_{j}^{n, l+1}-\frac{k_{j+1}}{k_{j}+k_{j+1}} \psi_{j+1}^{n, l+1}\right)_{\mid\left(0, a_{+}\right) \times \Gamma_{j}}
$$

and iterate until convergence.

\subsection{Finite dimensional approximation of the single layer problem}

We discretize equations (21)-(23) in space by a Galerkin method based on finite elements, and in age by finite differences. The initial condition is computed by a suitable quadrature rule. We outline the procedure for the single layer problem (19). Let then $\mathcal{T}_{h}$ be a triangulation of $\Omega$, namely $\Omega=\bigcup_{j=1}^{N} K_{j}$, where each $K_{j}=T_{K_{j}}(E) \in \mathcal{T}_{h}, E$ being the reference element, a simplex (namely the triangle with vertices $(0,0),(1,0)$, and $(0,1)$ when $d=2$ or the thetrahedron with vertices $(0,0,0),(1,0,0),(0,1,0)$, and $(0,0,1)$ when $d=3)$, or the unit cube $[0,1]^{d}$, and where $T_{K_{j}}$ is an invertible affine map. We define $h$ as the maximum diameter of the elements of the triangulation. The associated finite element spaces $X_{h}$ and $Y_{h}$ (see e.g. [15] for an introduction to finite element methods) are defined as

$$
X_{h}=\left\{v_{h} \in C^{0}(\Omega) \mid v_{h \mid K_{j}} \circ T_{K_{j}} \in \mathbb{P}_{1}(E)\right\}, Y_{h}=\left\{v_{h} \in C^{0}(\Omega) \mid v_{h \mid K_{j}} \circ T_{K_{j}} \in \mathbb{Q}_{1}(E)\right\},
$$

where $\mathbb{P}_{1}(E)$ is the space of polynomials of degree at most one on $E$, whereas $\mathbb{Q}_{1}(E)$ is the space of polynomials of degree at most one with respect to each variable on $E$. We denote by $\left\{\varphi_{j} \mid j=1, \ldots, N_{h}\right\}$ the usual nodal basis of the finite element space. 
A semi-discrete problem in space is obtained by applying a Galerkin procedure and choosing the finite dimensional space $V_{h}=X_{h}$, or $V_{h}=Y_{h}$, and a fully discrete approximation results from a backward Euler scheme in age. We let $a^{m}=m \Delta a\left(m=0,1, \ldots, N_{a}\right)$ be a partition of the age interval $\left[0, a_{\dagger}\right]$ into $N_{a}$ subintervals of amplitude $\Delta a=a_{\dagger} / N_{a}$, and we denote by $u_{h}^{n, m}$ the fully discrete approximation of $u\left(t^{n}, a^{m}, x\right)$. The numerical scheme for the single layer problem reads as follows.

Given $u_{h}^{0}$, for $n=1, \ldots, N_{t}$ :

$$
\left\{\begin{array}{l}
u_{h}^{n, 0}=\sum_{m=1}^{N_{a}} \Delta a\left[m\left(a^{m}\right) u_{h}^{n-1, m}\right] \\
\text { for } m=1, \ldots, N_{a}, \text { find } u_{h}^{n, m} \in V_{h} \text { such that } \\
\frac{\Delta t}{\Delta a}\left(u_{h}^{n, m}-u_{h}^{n, m-1}, v_{h}\right)+\Delta t A\left(a^{m} ; u_{h}^{n, m}, v_{h}\right)=\left(u_{h}^{n-1, m}, v_{h}\right) \quad \forall v_{h} \in V_{h}
\end{array}\right.
$$

Denoting by $U_{h}^{n}=\left(u_{h}^{n, 0}, u_{h}^{n, 1}, \ldots, u_{h}^{n, N_{a}}\right)$ the approximate solution at time $t=t^{n}$, we define the discrete $L^{1}\left(0, a_{\dagger} ; L^{2}(\Omega)\right)$ norm as

$$
\left\|U_{h}^{n}\right\|_{\mathcal{L}^{1}\left(0, a_{\dagger} ; L^{2}(\Omega)\right)}=\sum_{m=0}^{N_{a}} \Delta a\left\|u_{h}^{n, m}\right\|_{0},
$$

where $\|\cdot\|_{0}$ is the standard $L^{2}(\Omega)$ norm. Under some mild assumption on the exact solution, the following stability and convergence results for the scheme (24) hold (for proof we refer to [3]).

Proposition 4.1 ((Stability)) For any $n=1, \ldots, N_{t}$, the following estimate holds:

$$
\left\|U_{h}^{n}\right\|_{\mathcal{L}^{1}\left(0, a_{\dagger} ; L^{2}(\Omega)\right)} \leq\left(1+e^{a_{\dagger} \beta_{+}^{2} T}\right)\left\|U_{h}^{0}\right\|_{\mathcal{L}^{1}\left(0, a_{\dagger} ; L^{2}(\Omega)\right)} .
$$

where $\beta_{+}$is the one in (4).

Proposition 4.2 ((Convergence)) Let $\mathcal{T}_{h}$ be a regular family of triangulations on $\Omega$. Assume that the solution $u$ of the continuous problem is such that, for all $t \in(0, T), \frac{\partial u}{\partial a}(t, \cdot, \cdot), \frac{\partial u}{\partial t}(t, \cdot, \cdot) \in L^{1}\left(0, a_{\dagger} ; H^{1}(\Omega)\right)$, and $\frac{\partial^{2} u}{\partial a^{2}}(t, \cdot, \cdot), \frac{\partial^{2} u}{\partial t^{2}} \in L^{1}\left(0, a_{\dagger} ; L^{2}(\Omega)\right)$. Then, using linear finite elements, the following estimate holds

$$
\begin{aligned}
& \left\|u\left(t^{n}, \cdot, \cdot\right)-U_{h}^{n}\right\|_{\mathcal{L}^{1}\left(0, a_{\dagger} ; L^{2}(\Omega)\right)} \leq\left\|U_{h}^{0}-\Pi_{h} u_{0}\right\|_{\mathcal{L}^{1}\left(0, a_{\dagger} ; L^{2}(\Omega)\right)}+C h\left\|u\left(t^{n}, \cdot, \cdot\right)\right\|_{\mathcal{L}^{1}\left(0, a_{\dagger} ; H^{1}(\Omega)\right)} \\
& \quad+C h \sum_{p=0}^{n} \Delta t\left\|\frac{\partial u}{\partial a}\left(t^{p}, \cdot, \cdot\right)\right\|_{L^{1}\left(0, a_{\dagger} ; H^{1}(\Omega)\right)}+C h \int_{0}^{t^{n}}\left\|\frac{\partial u}{\partial t}(t, \cdot, \cdot)\right\|_{\mathcal{L}^{1}\left(0, a_{\dagger} ; H^{1}(\Omega)\right)} d t \\
& \quad+C \Delta t \int_{0}^{t^{n}}\left\|\frac{\partial^{2} u}{\partial t^{2}}(t, \cdot, \cdot)\right\|_{\mathcal{L}^{1}\left(0, a_{\dagger} ; L^{2}(\Omega)\right)} d t+C \Delta a \sum_{p=0}^{n} \Delta t\left\|\frac{\partial^{2} u}{\partial a^{2}}\left(t^{p}, \cdot, \cdot\right)\right\|_{L^{1}\left(0, a_{\dagger} ; L^{2}(\Omega)\right)}
\end{aligned}
$$

where the constant $C>0$ is independent of $h, \Delta a$, and $\Delta t$.

\subsection{Finite dimensional approximation of the multi-layer problem}

We focus on the time slab $\left(t^{n-1}, t^{n}\right)$. The matrix associated to problems (21)-(22), at age $a^{m}$, is given in $\Omega_{j}$ by

$$
A_{j}\left(a^{m}\right)=\Delta t \Delta a S^{j}\left(a^{m}\right)+(\Delta t+\Delta a) M^{j},
$$


where $S^{j}\left(a^{m}\right)$ and $M^{j}$ are the local stiffness and mass matrices, respectively, defined as

$$
S_{p q}^{j}\left(a^{m}\right)=\int_{\Omega_{j}} k\left(a^{m}\right) \nabla \varphi_{q} \nabla \varphi_{p} d x \quad M_{p q}^{j}=\int_{\Omega_{j}} \varphi_{p} \varphi_{q} d x
$$

If we decompose the local degrees of freedom in internal and interface degrees of freedom, the matrix $A_{j}\left(a^{m}\right)$ can be decomposed into

$$
A_{j}\left(a^{m}\right)=\left[\begin{array}{cc}
A_{j}^{0}\left(a^{m}\right) & B_{j}\left(a^{m}\right) \\
\tilde{B}_{j}^{T}\left(a^{m}\right) & \bar{A}_{j}\left(a^{m}\right)
\end{array}\right]
$$

where $A_{j}^{0}$ is the block corresponding to the interaction between internal points, $\bar{A}_{j}$ is the block corresponding to the interaction between interface points, whereas $B_{j}$ and $\tilde{B}_{j}^{T}$ are the ones corresponding to the interaction between internal and interface points. As the spatial domain is decomposed into strips, after a suitable reordering of the unknowns, the solution at age $a^{m}$ is $\mathbf{u}^{n, m}=\left(\boldsymbol{u}_{1}^{n, m}, \boldsymbol{\lambda}_{1}^{n, m}, \boldsymbol{u}_{2}^{n, m}, \ldots, \boldsymbol{u}_{N-1}^{n, m}, \boldsymbol{\lambda}_{N-1}^{n, m}, \boldsymbol{u}_{N}^{n, m}\right)^{T}$, while the forcing term is $\mathbf{f}^{n, m}=\left(\boldsymbol{f}_{1}^{m}, \boldsymbol{f}_{\Gamma_{1}}^{m}, \boldsymbol{f}_{2}^{m}, \ldots, \boldsymbol{f}_{N-1}^{m}, \boldsymbol{f}_{\Gamma_{N-1}}^{m}, \boldsymbol{f}_{N}^{m}\right)^{T}$, where we have denoted by $\boldsymbol{u}_{j}^{m}$ and $\boldsymbol{f}_{j}^{m}=\Delta t M^{j} \boldsymbol{u}_{j}^{n, m-1}+$ $\Delta a M^{j} \boldsymbol{u}_{j}^{n-1, m}$ the restrictions of the solution and the forcing term to $\Omega_{j}$, and by $\boldsymbol{\lambda}_{j}^{n, m}$ and $\boldsymbol{f}_{\Gamma_{j}}^{m}$ the trace on the interface $\Gamma_{j}$ of the solution and the forcing term at age $a^{m}$, respectively. The coupled problem at age $a^{m}$ is then given by

$$
\mathcal{A}\left(a^{m}\right) \mathbf{u}^{n, m}=\mathbf{f}^{n, m},
$$

where

$$
\mathcal{A}\left(a^{m}\right)=\left[\begin{array}{ccccccc}
A_{11}^{m} & A_{1 \Gamma_{1}}^{m} & & & & & \\
A_{\Gamma_{1} 1}^{m} & A_{\Gamma_{1} \Gamma_{1}}^{m} & A_{2 \Gamma_{1}}^{m} & & & & \\
& A_{\Gamma_{1} 2}^{m} & A_{22}^{m} & A_{2 \Gamma_{2}}^{m} & & \\
& & \ddots & \ddots & \ddots & & \\
& & & A_{\Gamma_{N-2} N-1}^{m} & A_{N-1 N-1} & A_{N-1 \Gamma_{N-1}}^{m} & \\
& & & & A_{\Gamma_{N-1} N-1}^{m} & A_{\Gamma_{N-1} \Gamma_{N-1}}^{m} & A_{N \Gamma_{N-1}}^{m} \\
& & & & & A_{\Gamma_{N-1} N}^{m} & A_{N N}^{m}
\end{array}\right],
$$

where we have set $A_{i j}^{m}=A_{i j}\left(a^{m}\right)$. By splitting the interface matrices

$$
A_{\Gamma_{j} \Gamma_{j}}\left(a^{m}\right)=A_{\Gamma_{j} \Gamma_{j}}^{(j)}\left(a^{m}\right)+A_{\Gamma_{j} \Gamma_{j}}^{(j+1)}\left(a^{m}\right),
$$

where $A_{\Gamma_{j} \Gamma_{j}}^{(i)}\left(a^{m}\right)$ is the contribution to $A_{\Gamma_{j} \Gamma_{j}}\left(a^{m}\right)$ coming from the domain $\Omega_{i}(i=j, j+1)$, the matrices associated to the restriction of the bilinear form $A_{j}\left(a^{m} ; \cdot, \cdot\right)$ to domain $\Omega_{j}$ at age $a^{m}$, corresponding to the discretization of the local Neumann problems, are given by

$$
A_{j}^{m}=\left[\begin{array}{ccc}
A_{\Gamma_{j-1} \Gamma_{j-1}}^{(j), m} & A_{j \Gamma_{j-1}}^{m} & 0 \\
A_{\Gamma_{j-1} j}^{m} & A_{j j}^{m} & A_{j \Gamma_{j}}^{m} \\
0 & A_{\Gamma_{j} j}^{m} & A_{\Gamma_{j} \Gamma_{j}}^{(j), m}
\end{array}\right]
$$

for $j=2, \ldots, N$, and

$$
A_{1}^{m}=\left[\begin{array}{cc}
A_{11}^{m} & A_{1 \Gamma_{1}}^{m} \\
A_{\Gamma_{1} 1}^{m} & A_{\Gamma_{1} \Gamma_{1}}^{(1, m}
\end{array}\right] \quad A_{N}^{m}=\left[\begin{array}{cc}
A_{\Gamma_{N-1} \Gamma_{N-1}}^{(N), m} & A_{N \Gamma_{N-1}} \\
A_{\Gamma_{N-1} N} & A_{N N}
\end{array}\right] .
$$


Aside, the matrices associated to the local Dirichlet problem are

$$
D_{j}^{m}=\left[\begin{array}{ccc}
I d_{\Gamma_{j-1}} & 0 & 0 \\
A_{\Gamma_{j-1}}^{m} & A_{j j}^{m} & A_{j \Gamma_{j}}^{m} \\
0 & 0 & I d_{\Gamma_{j}}
\end{array}\right],
$$

for $j=2, \ldots, N$, and

$$
D_{1}^{m}=\left[\begin{array}{cc}
A_{11}^{m} & A_{1 \Gamma_{1}}^{m} \\
0 & I d_{\Gamma_{1}}
\end{array}\right] \quad D_{N}^{m}=\left[\begin{array}{cc}
I d_{\Gamma_{N-1}} & 0 \\
A_{N \Gamma_{N-1}} & A_{N N}
\end{array}\right] .
$$

We drop hereafter any index referring to time discretization, in order to improve readability. The discrete waveform relaxation algorithm then reads, inside each time step, as follows.

Given $\boldsymbol{\lambda}^{0}=\left(\boldsymbol{\lambda}^{1,0}, \ldots, \boldsymbol{\lambda}^{N_{a}, 0}\right) \in \Lambda^{N_{a}}$, for $k \geq 1$

- Solve, for $m=1, \ldots, N_{a}$ :

$$
\begin{cases}A_{11}^{m} \boldsymbol{u}_{1}^{m, l+1} & =\boldsymbol{f}_{1}^{m}-A_{1 \Gamma_{1}}^{m} \boldsymbol{\lambda}_{1}^{m, l} \\ A_{j j}^{m} \boldsymbol{u}_{j}^{m, l+1} & =\boldsymbol{f}_{j}^{m}-A_{j \Gamma_{j}}^{m} \boldsymbol{\lambda}_{j-1}^{m, l}-A_{\Gamma_{j-1} j}^{m} \boldsymbol{\lambda}_{j}^{m, l} \quad j=2, \ldots, N \\ A_{N N}^{m} \boldsymbol{u}_{N}^{m, l+1} & =\boldsymbol{f}_{N}^{m}-A_{N \Gamma_{N-1}} \lambda_{N-1}^{m, l}\end{cases}
$$

- Solve, for $m=1, \ldots, N_{a}$ :

$$
\begin{aligned}
& {\left[\begin{array}{cc}
A_{11}^{m} & A_{1 \Gamma_{1}}^{m} \\
A_{\Gamma_{1} 1}^{m} & A_{\Gamma_{1} \Gamma_{1}}^{(1), m}
\end{array}\right]\left[\begin{array}{c}
\boldsymbol{\psi}_{1}^{m, l+1} \\
\boldsymbol{\mu}_{1,1}^{m, l+1}
\end{array}\right]=} \\
& =\left[\begin{array}{c}
\mathbf{0} \\
\boldsymbol{f}_{\Gamma 1}^{m}+A_{\Gamma_{1} 1}^{m} \boldsymbol{u}_{1}^{m, l+1}+A_{\Gamma_{1} \Gamma_{1}}^{(1), m} \boldsymbol{\lambda}_{1}^{m, l}+A_{2 \Gamma_{1}}^{m} \boldsymbol{u}_{2}^{m, l+1}+A_{\Gamma_{1} \Gamma_{1}}^{(2), m} \boldsymbol{\lambda}_{1}^{m, l}
\end{array}\right], \\
& {\left[\begin{array}{ccc}
A_{\Gamma_{j-1} \Gamma_{j-1}}^{(j), m} & A_{j \Gamma_{j-1}}^{m} & 0 \\
A_{\Gamma_{j-1} j}^{m} & A_{j j}^{m} & A_{j \Gamma_{j}}^{m} \\
0 & A_{\Gamma_{j} j}^{m} & A_{\Gamma_{j} \Gamma_{j}}^{(j), m}
\end{array}\right]\left[\begin{array}{c}
\boldsymbol{\mu}_{j, j-1}^{m, l+1} \\
\boldsymbol{\psi}_{j}^{m, l+1} \\
\boldsymbol{\mu}_{j, j}^{m, l+1}
\end{array}\right]=} \\
& =\left[\begin{array}{c}
\boldsymbol{f}_{\Gamma_{j-1}}^{m}-A_{\Gamma_{j-1} j}^{m} \boldsymbol{u}_{j-1}^{m, l+1}-A_{\Gamma_{j-1} \Gamma_{j-1}}^{(j-1), m} \boldsymbol{\lambda}_{j-1}^{m, l}-A_{j \Gamma_{j}}^{m} \boldsymbol{u}_{j}^{m, l+1}-A_{\Gamma_{j} \Gamma_{j}}^{(j), m} \boldsymbol{\lambda}_{j}^{m, l} \\
\mathbf{0} \\
\boldsymbol{f}_{\Gamma_{j}}^{m}+A_{\Gamma_{j j} j}^{m} \boldsymbol{u}_{j}^{m, l+1}+A_{\Gamma_{j} \Gamma_{j}}^{(j), m} \boldsymbol{\lambda}_{j}^{m, l}+A_{j \Gamma_{j}}^{m} \boldsymbol{u}_{j+1}^{m, l+1}+A_{\Gamma_{j} \Gamma_{j}}^{(j+1), m} \boldsymbol{\lambda}_{j+1}^{m, l}
\end{array}\right],
\end{aligned}
$$




$$
\begin{aligned}
& {\left[\begin{array}{cc}
A_{\Gamma_{N-1} \Gamma_{N-1}}^{(N), m} & A_{N \Gamma_{N-1}} \\
A_{\Gamma_{N-1} N} & A_{N N}
\end{array}\right]\left[\begin{array}{c}
\boldsymbol{\mu}_{N, N-1}^{m, l+1} \\
\boldsymbol{\psi}_{N}^{m, l+1}
\end{array}\right]=} \\
& \quad=\left[\begin{array}{cc}
\boldsymbol{f}_{\Gamma_{N-1}}^{m}+A_{\Gamma_{N-1} N}^{m} \boldsymbol{u}_{N-1}^{m, l+1}-A_{\Gamma_{1} \Gamma_{1}}^{(N-1), m} \boldsymbol{\lambda}_{N-1}^{m, l}-A_{N \Gamma_{N-1}}^{m} \boldsymbol{u}_{N}^{m, l+1}-A_{\Gamma_{1} \Gamma_{1}}^{(N), m} \boldsymbol{\lambda}_{N-1}^{m, l} \\
\mathbf{0}
\end{array}\right] .
\end{aligned}
$$

- Set, for $m=1, \ldots, N_{a}$ :

$$
\boldsymbol{\lambda}_{j}^{m, l+1}=\boldsymbol{\lambda}_{j}^{m, l}-\theta\left[\frac{k_{j}}{k_{j}+k_{j+1}} \boldsymbol{\psi}_{j}^{m, l+1}+\frac{k_{j+1}}{k_{j}+k_{j+1}} \boldsymbol{\psi}_{j+1}^{m, l+1}\right], \quad j=1, \ldots, N-1,
$$

and iterate until convergence.

\section{$5 \quad$ Numerical Results}

In this section we provide some numerical tests to show the effectiveness of the method. We consider diffusion coefficients that are uniform in age and heterogeneous in space, with the ratio $\delta k=k_{1} / k_{2}$ up to $10^{4}$. The numerical simulations are run on Matlab ${ }^{\circledR} 6.5$.
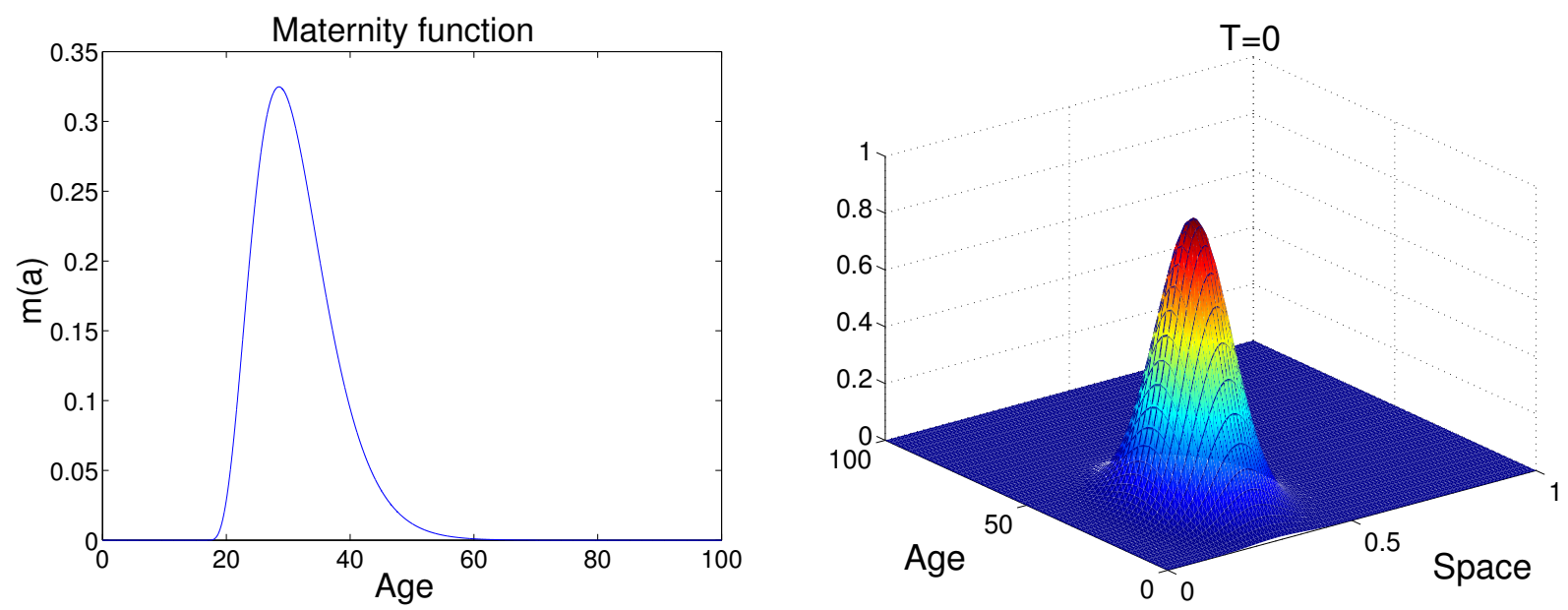

Figure 3: Maternity function (left) and initial age-space profile (right) for the numerical tests

\subsection{A two-domains problem}

In this first series of tests we consider a population spreading in a one dimensional environment constituted of two layers. We solve problem (20) on the domain $\Omega=[0,1]$, and we assume $a_{\dagger}=100$ as maximal age. We choose $\Delta a=2$, as well as $\Delta t=1$. We let $\Omega=\Omega_{1} \cup \Omega_{2}$, with $\Omega_{1}=(0, \alpha), \Omega_{2}=(\alpha, 1)$, and we discretize problem (20) in space via $\mathbb{P}_{1}$ finite elements. We use an uniform mesh in space, with $h_{1}=h_{2}=1 / 100$, and, at each time level, we use in (24) a Simpson quadrature rule over two adjacent subdomains to compute the intial value 


\begin{tabular}{|c|c|c|c|c|c|c|c|}
\hline$\delta k$ & $T=1$ & $T=3$ & $T=6$ & $T=9$ & $T=12$ & $T=15$ & $T=20$ \\
\hline \hline 1 & 13 & 11 & 11 & 10 & 10 & 10 & 10 \\
\hline 10 & 17 & 15 & 14 & 14 & 14 & 14 & 13 \\
\hline $10^{2}$ & 23 & 20 & 20 & 20 & 19 & 19 & 19 \\
\hline $10^{3}$ & 26 & 23 & 23 & 23 & 22 & 22 & 22 \\
\hline $10^{4}$ & 32 & 27 & 27 & 26 & 26 & 25 & 25 \\
\hline
\end{tabular}

Table 1: Two subdomains, $\alpha=0.5$ : iteration counts per time step, $\left\|\boldsymbol{\lambda}^{k+1}-\boldsymbol{\lambda}^{k}\right\|_{\Lambda} /\left\|\boldsymbol{\lambda}^{k}\right\|_{\Lambda}<10^{-6}$.

for the parabolic (in age and space) problem. We consider a non-symmetric initial distribution of population (with respect to both space and age) given by

$$
u_{0}(x, a)=e^{-\left(\frac{(a-30)^{2}}{200}+100(x-0.4)^{2}\right)},
$$

and we take the mortality and fertility function as

$$
\mu(a)=\frac{1}{a_{\dagger}-a}, \quad \beta(a)= \begin{cases}0 & \text { if } a \leq a_{1} \\ \frac{\beta\left(a-a_{1}\right)^{\alpha-1} e^{-\frac{\left(a-a_{1}\right)}{\vartheta}}}{\vartheta^{\alpha} \Gamma(\alpha)} & \text { if } a_{1}<a<a_{2} \\ 0 & \text { if } a \geq a_{2},\end{cases}
$$

where we set $a_{1}=17, a_{2}=70, \beta=7, \alpha=5$, and $\vartheta=3$.

We plot in Figure 3 the resulting maternity function and the initial profile of the problem.
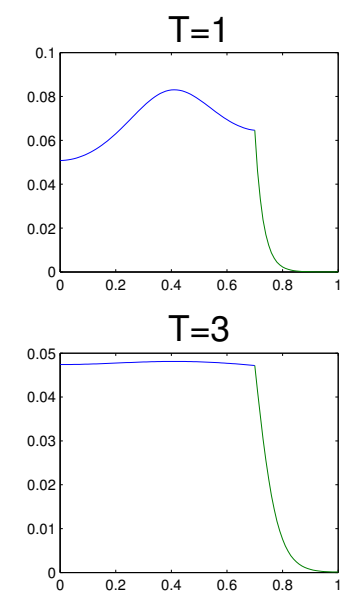

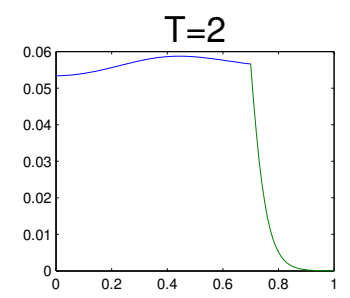

$\mathrm{T}=5$

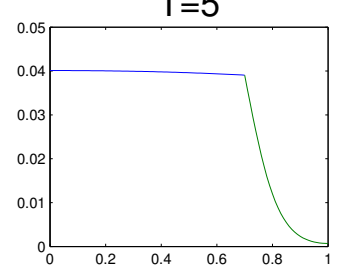

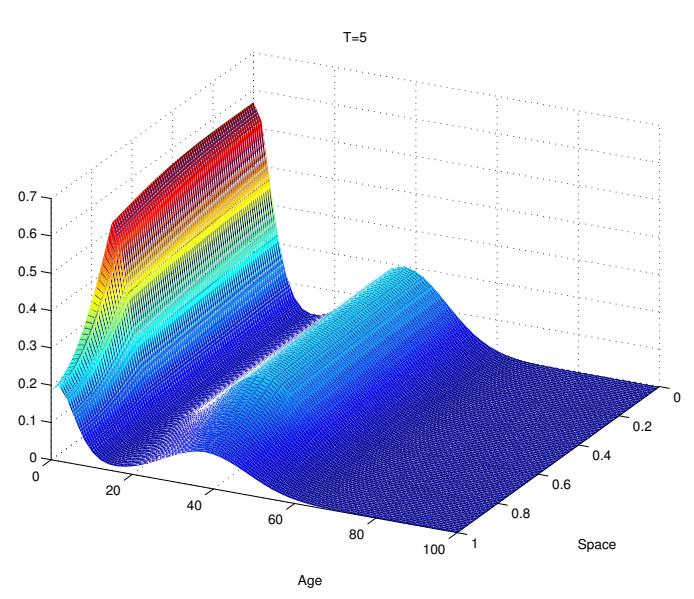

Figure 4: Time evolution of the profile at age $a=20$ (left), and age-space profile at time $T=5$ (right), $\delta k=100$.

In Table 1 and 2 we report the iteration counts at different time levels for two different positions of the interface, $\alpha=0.5$ and $\alpha=0.7$. The stopping criterion is given by $\left\|\boldsymbol{\lambda}^{k+1}-\boldsymbol{\lambda}^{k}\right\|_{\Lambda} /\left\|\boldsymbol{\lambda}^{k}\right\|_{\Lambda}<10^{-6}$. The number of iterations increases with the amplitude of the jumps in the diffusive coefficients, but the algorithm appears to be robust with respect to the position of the interface and with respect to the evolution in time. In Figure 2 we report the time evolution of the space profile of individuals of age 20, and the age-space profile of the solution at time $T=5$, with $\delta k=100$. The jump in the normal derivative due to the high heterogenity of the spatial medium 


\begin{tabular}{|c|c|c|c|c|c|c|c|}
\hline$\delta k$ & $T=1$ & $T=3$ & $T=6$ & $T=9$ & $T=12$ & $T=15$ & $T=20$ \\
\hline \hline 1 & 17 & 11 & 11 & 10 & 10 & 10 & 10 \\
\hline 10 & 22 & 14 & 14 & 14 & 14 & 13 & 13 \\
\hline $10^{2}$ & 32 & 20 & 19 & 19 & 19 & 18 & 18 \\
\hline $10^{3}$ & 37 & 23 & 22 & 22 & 22 & 21 & 21 \\
\hline $10^{4}$ & 44 & 26 & 26 & 25 & 25 & 25 & 25 \\
\hline
\end{tabular}

Table 2: Two subdomains, $\alpha=0.7$ : iteration counts per time level, $\left\|\boldsymbol{\lambda}^{k+1}-\boldsymbol{\lambda}^{k}\right\|_{\Lambda} /\left\|\boldsymbol{\lambda}^{k}\right\|_{\Lambda}<10^{-6}$.

is clearly visible.

\subsection{Influence of the number of subdomains}

In this series of tests we analyze the scalability of the method with respect to the number of subdomains. We consider the domain $\Omega=(0,1)$ decomposed into $2,4,8$, and 10 subdomains of equal size, and we consider a black and white coloring, where each subdomain is surrounded by subdomains with different diffusion coefficients. The mesh size in space is the same for all subdomains $(h=1 / 100)$, the time and age step are $\Delta t=1$, and $\Delta a=2$. We give in Table 3 through 5 the iteration counts at different time levels for decompositions into 4,8 , and 10 subdomains, with different ratios between the diffusion coefficients. The number of iterations shows the same pattern observed in the two-domains decomposition: it increases with the ratio of the diffusion coefficients but it remains reasonable, and it shows robustness with respect to the evolution in time.

We then focused on scalability, chosing $\delta k=100$. We report in Table 6 the iteration counts with respect to the subdomain decomposition. Evidence is an increase with the number of subdomains, albeit the algorithm appears to be stable with respect to the evolution in time, see Figure 5 and 6 .

Two comments are in order. On the one hand, in all the simulations the number of iterations needed to solve the coupled problem at the first time level is higher (significantly in some cases) than the number of iterations needed to solve the coupled problem at subsequent time levels. This is quite unsurprising, and it is a consequence of the kind of problem we are dealing with. Indeed, the initial condition is constituted only of individuals older that a certain age, whereas at time $T=1$ offsprings appear where no individual was present earlier and the algorithm has to deal with it. This is a peculiar feature of the first time level, which no longer reproduces in the rest of the computation, owing to the smoothing properties of the parabolic problem. On the other hand, the increase in the average number of iterations per time step is significant with the increase of the number of subdomains, thus the introduction of a suitable coarse space becomes mandatory, and it is currently under investigation. Aside, we plot in Figure 7 (left) the time evolution of the space profile of individuals of age

\begin{tabular}{|c|c|c|c|c|c|c|c|}
\hline$\delta k$ & $T=1$ & $T=3$ & $T=6$ & $T=9$ & $T=12$ & $T=15$ & $T=20$ \\
\hline \hline 1 & 22 & 21 & 20 & 19 & 19 & 19 & 19 \\
\hline 10 & 32 & 28 & 27 & 26 & 25 & 25 & 24 \\
\hline $10^{2}$ & 43 & 37 & 36 & 35 & 34 & 34 & 33 \\
\hline $10^{3}$ & 45 & 38 & 37 & 37 & 36 & 36 & 35 \\
\hline $10^{4}$ & 45 & 39 & 38 & 37 & 37 & 36 & 35 \\
\hline
\end{tabular}

Table 3: Four subdomains: iteration counts per time step, $\left\|\boldsymbol{\lambda}^{k+1}-\boldsymbol{\lambda}^{k}\right\|_{\Lambda} /\left\|\boldsymbol{\lambda}^{k}\right\|_{\Lambda}<10^{-6}$.

20 in the four domains subdivision case. Again, the jump in the normal derivative consequence of the high heterogeneity of the medium is clearly appreciable. In Figure 7 (right) we also plot the traces of the interface 


\begin{tabular}{|c|c|c|c|c|c|c|c|}
\hline$\delta k$ & $T=1$ & $T=3$ & $T=6$ & $T=9$ & $T=12$ & $T=15$ & $T=20$ \\
\hline \hline 1 & 39 & 36 & 34 & 33 & 33 & 32 & 31 \\
\hline 10 & 52 & 46 & 44 & 43 & 43 & 42 & 40 \\
\hline $10^{2}$ & 69 & 62 & 59 & 58 & 57 & 56 & 54 \\
\hline $10^{3}$ & 83 & 73 & 71 & 70 & 69 & 68 & 66 \\
\hline $10^{4}$ & 85 & 76 & 74 & 72 & 71 & 70 & 69 \\
\hline
\end{tabular}

Table 4: Eight subdomains: iteration counts per time step, $\left\|\boldsymbol{\lambda}^{k+1}-\boldsymbol{\lambda}^{k}\right\|_{\Lambda} /\left\|\boldsymbol{\lambda}^{k}\right\|_{\Lambda}<10^{-6}$.

\begin{tabular}{|c|c|c|c|c|c|c|c|}
\hline$\delta k$ & $T=1$ & $T=3$ & $T=6$ & $T=9$ & $T=12$ & $T=15$ & $T=20$ \\
\hline \hline 1 & 51 & 50 & 48 & 47 & 46 & 45 & 44 \\
\hline 10 & 67 & 66 & 63 & 61 & 60 & 59 & 58 \\
\hline $10^{2}$ & 90 & 88 & 84 & 82 & 81 & 79 & 77 \\
\hline $10^{3}$ & 111 & 108 & 104 & 102 & 101 & 99 & 97 \\
\hline $10^{4}$ & 116 & 112 & 108 & 106 & 105 & 103 & 101 \\
\hline
\end{tabular}

Table 5: Ten subdomains: iteration counts per time step, $\left\|\boldsymbol{\lambda}^{k+1}-\boldsymbol{\lambda}^{k}\right\|_{\Lambda} /\left\|\boldsymbol{\lambda}^{k}\right\|_{\Lambda}<10^{-6}$.

\begin{tabular}{|c|c|c|c|c|c|c|c|}
\hline SD & $T=1$ & $T=3$ & $T=6$ & $T=9$ & $T=12$ & $T=15$ & $T=20$ \\
\hline \hline 2 & 23 & 20 & 19 & 19 & 19 & 18 & 18 \\
\hline 4 & 43 & 37 & 36 & 35 & 34 & 34 & 33 \\
\hline 8 & 69 & 62 & 59 & 58 & 57 & 56 & 54 \\
\hline 10 & 90 & 88 & 84 & 82 & 81 & 79 & 77 \\
\hline
\end{tabular}

Table 6: Iteration counts per time level for different subdomain decompositions: $<10^{-6}, \delta k=10^{2}$.
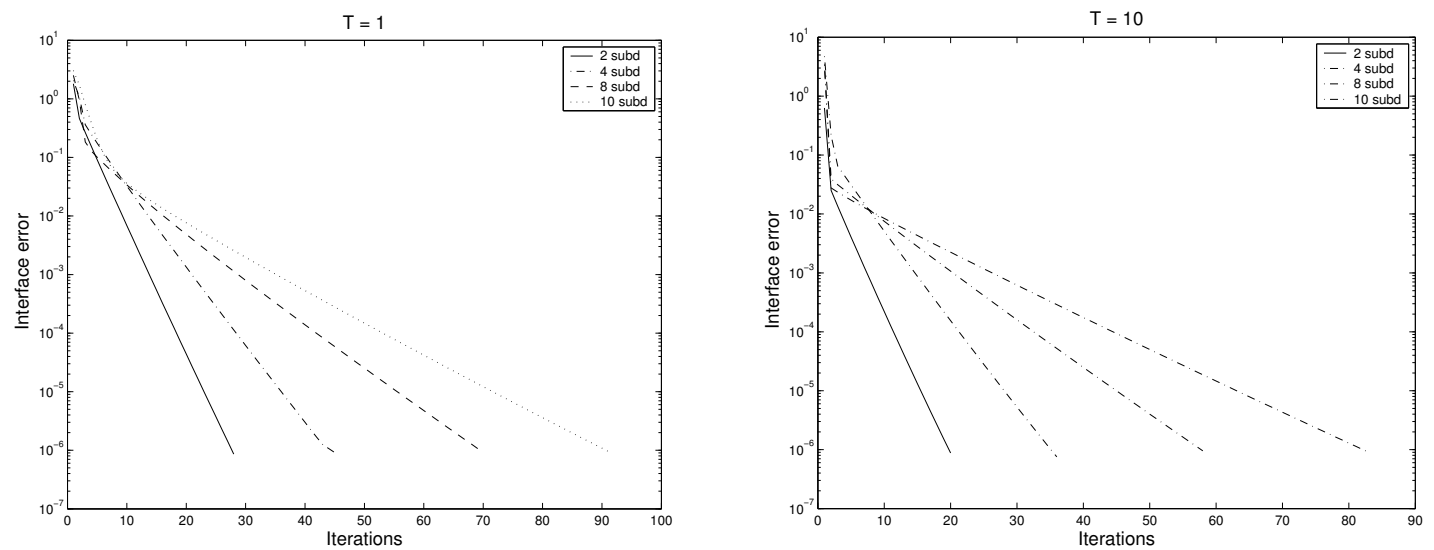

Figure 5: Convergence history with respect to the number of subdomains at different time steps: $T=1$ (left) and $T=10$ (right), $\delta k=100$.

variables and the space profile of individuals of age 20 at convergence after a longer computation $(T=30)$ for 

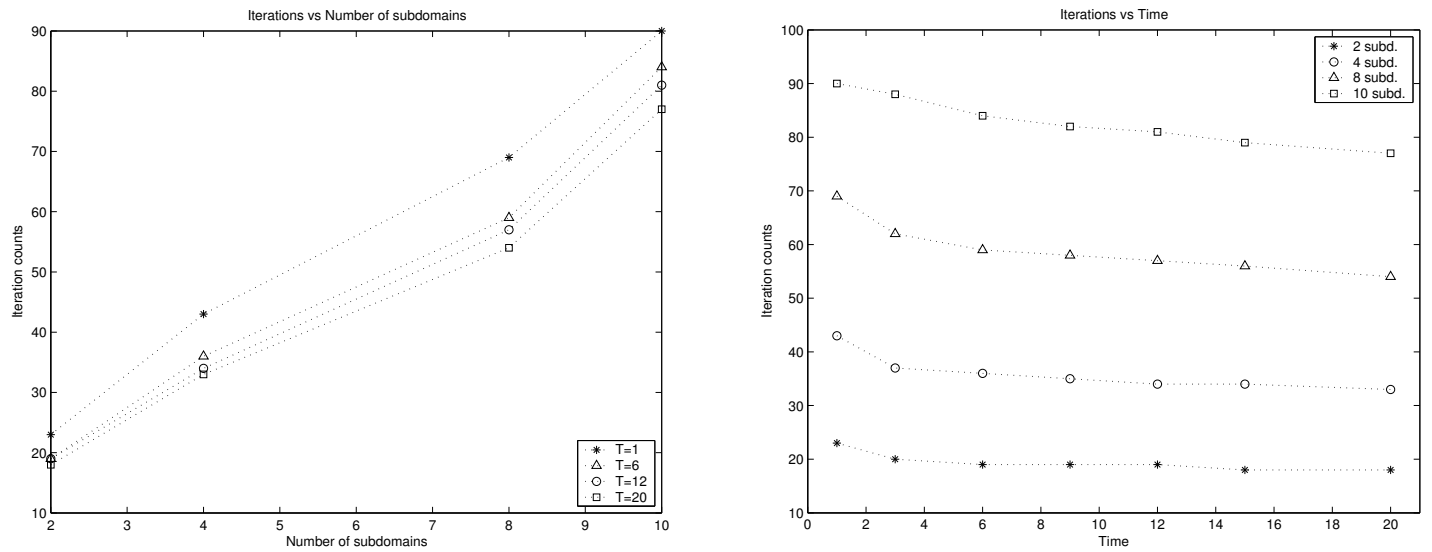

Figure 6: Iteration counts vs the number of subdomains at different time levels, $\delta k=100$.

the eight domains subdivision case: the interface variables show the typical age profile resulting from the linear problem we are considering.
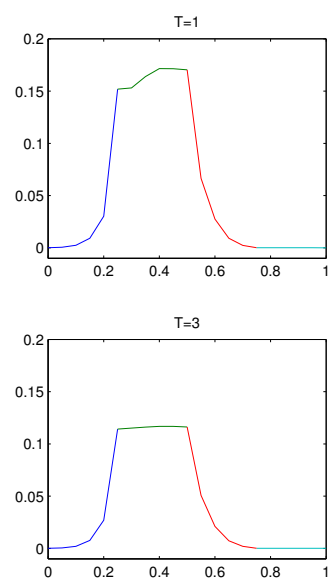

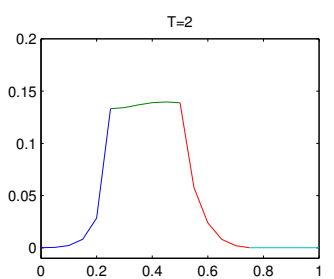

$=5$

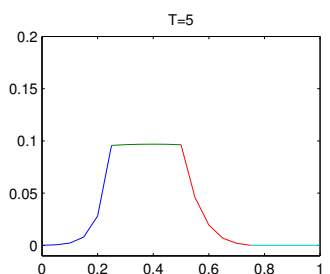

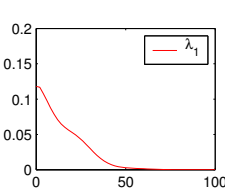
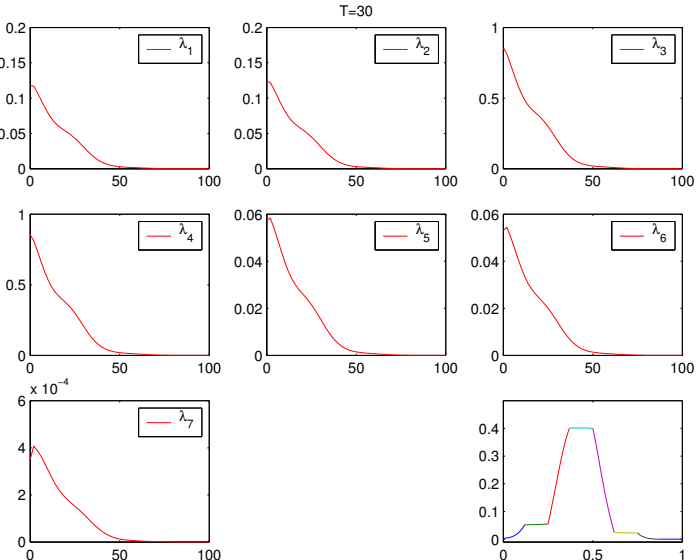

Figure 7: Left: time evolution of the profile at age $a=20$ for a decomposition into 4 subdomains $(\delta k=$ 100). Right: interface variables and profile of the solution (in $a=20$ ), at convergence at time $T=30$ for a decomposition into 8 subdomains $(\delta k=100)$.

\section{Conclusions}

We propose here a generalization of the balancing Neumann-Neumann algorithm, devised for purely elliptic problems, to approximate the solution of the diffusion of an age-structured population in a multilayer environment. The proposed algorithm appears to be robust in terms of iteration counts with respect to the heterogeneities in the viscosity coefficients, and the long time simulation. As the performance of the method degradates with the number of subdomains, further investigations will focus, on the one hand, on the development of a suitable coarse space to speed up the convergence in the multidomain situation, and on the testing of Optimized Schwarz 
Waveform Relaxation (recently proposed for advection-diffusion-reaction equations by M.J. Gander at al. [6]), to such framework. On the other hand such approach will be extended to the numerical approximation of a nonlinear model, where the nonlinearity is located not only in the diffusion coefficients, but also in the fertility or mortality functions, that can depend upon the total population. Finally, the algorithm will be tested on more complex situations of practical interest.

\section{References}

[1] B.P. Ayati and T. Dupont. Galerkin methods in age and space for a population model with nonlinear diffusion. SIAM J. Num. Anal., 40(3):1064-1076, 2002.

[2] C. Cusulin and L. Gerardo-Giorda. A domain decomposition method for the diffusion of an age-structured population in a multilayer environment. In Domain Decomposition Methods in Science and Engineering (Proceedings of the DD17 Conference), pages 461-468. Springer-Verlag, 2007.

[3] C. Cusulin and L. Gerardo-Giorda. A FEM-Galerkin approximation for diffusion in age-structured population dynamics. Technical report, HAL - Hyper Article on Line, 2007. http://hal.archives-ouvertes.fr/hal00183067 - submitted.

[4] C. Cusulin, M. Iannelli, and G. Marinoschi. Age-structured diffusione in a multi-layer environment. Nonlinear Analysis: Real World Applications, 6(1):207-223, 2006.

[5] C. Cusulin, M. Iannelli, and G. Marinoschi. Convergence in a multi-layer population model with agestructure. Nonlinear Analysis: Real World Applications, 8:887-902, 2007.

[6] M.J. Gander and L. Halpern. Optimized Schwarz Waveform Relaxation for advection reaction diffusion problems. SIAM Journal on Numerical Analysis, 45(2):666-697,, 2007.

[7] M.-Y. Kim. Galerkin methods for a model of population dynamics with nonlinear diffusion. Num. Meth. Partial Differential Equations, 12:59-73, 1996.

[8] M.-Y. Kim and E.-J. Park. Characteristic finite element methods for diffusion epidemic models with age-structured populations. Comput. Math. Appl., 97:55-70, 1998.

[9] N. Kinezaki, K. Kawasaki, F. Takasu, and N. Shigesada. Modeling biological invasion into periodically fragmented environments. Theor. Popul. Biol., 64:291-302, 2003.

[10] P. Le Tallec and M. Vidrascu. Generalized Neumann-Neumann preconditioners for iterative substructuring. In Petter E. Bjørstad, Magne Espedal, and David Keyes, editors, Domain Decomposition Methods in Sciences and Engineering. John Wiley \& Sons, 1997. Proceedings from the Ninth International Conference, June 1996, Bergen, Norway.

[11] J.-L. Lions and E. Magenes. Non-Homogeneous Boundary Value Problems. Springer-Verlag, New YorkBerlin - Heidelberg, 1972.

[12] F.A. Milner. A numerical method for a model of population dynamics with spatial diffusion. Comp. Math Appl., 19(31), 1990.

[13] A. Okubo and S. A. Levin. Diffusion and ecological problems: modern perspectives. Springer, New York, 2001.

[14] A. Okubo and S.A. Levin. Diffusion and ecological problems: modern perspectives. Springer, New York, 2001. 
[15] A. Quarteroni and A. Valli. Numerical Approximation of Partial Differential Equations. Springer-Verlag, Berlin, 1994.

[16] A. Quarteroni and A. Valli. Domain Decompostion Methods for Partial Differential Equations. Oxford University Press, 1999.

[17] N. Shigesada and K. Kawasaki. Biological invasions: theory and practice. Oxford University Press, New York, 1997. 